\title{
The Landau band effects in the quantum magnetic oscillations and the deviations from the quasiclassical Lifshitz-Kosevich theory in quasi-two-dimensional conductors
}

\author{
V.M. Gvozdikov \\ Kharkov National University, Kharkov 61077, Ukraine \\ Max-Planck-Institut für Physik Komplexer Systeme, 38 Nöthnitzer Strasse, D-01187 Dresden, Germany \\ E-mail: vladimir.gvozdikov@gmail.com
}

Received May 24, 2011

\begin{abstract}
The quantum magnetic oscillations (QMO) in the layered and quasi-two-dimensional (2D) conductors deviate from the quasiclassical Lifshitz-Kosevich (LK) theory developed for 3D conventional metals. We discuss deviations related to the broadening of the Landau levels into Landau bands by various mechanisms (layer-stacking, magnetic breakdown, incoherence, disorder, localization etc.). Each mechanism yields a specific factor modulating the QMO amplitudes depending on the density of states and electron velocities within the Landau bands. In contrast to the LK theory, these factors differ for the thermodynamic (de Haas-van Alphen (dHvA)) and kinetic (Shubnikov-de Haas $(\mathrm{SdH})$ ) oscillations. We calculated the magnetic breakdown damping factors for the $\mathrm{SdH}$ and $\mathrm{dHvA}$ oscillations in the 2D conductors and analyzed their difference as well as the analogy between the bandwidth and Weiss oscillations. In case of an isotropic 3D metals the kinetic factors become proportional to the thermodynamic ones as is assumed in the LK theory.
\end{abstract}

PACS: 71.18.+y Fermi surface: calculations and measurements; effective mass, g factor;

72.15.Gd Galvanomagnetic and other magnetotransport effects;

73.50.Jt Galvanomagnetic and other magnetotransport effects (including thermomagnetic effects).

Keywords: quantum magnetic oscillations, quasi-two-dimensional conductors, magnetic breakdown, damping factors.

\section{Introduction}

The quantum magnetic oscillations (QMO), the de Haas-van Alphen (dHvA) and Shubnikov-de Haas ( $\mathrm{SdH})$ oscillations, are known as the most powerful experimental tools for studies of the Fermi surfaces (FS) in metals. A theoretical basis for these studies was laid down in a seminal Lifshitz-Kosevich (LK) paper [1]. Details of the method based on the LK theory can be found in the textbooks $[2,3]$. The important advantage of this method is that it permits to extract the values of effective electron masses, scattering times, gyromagnetic factors for different crosssections of the FS directly from the experimental data on the $\mathrm{SdH}$ and dHvA oscillations.

The LK theory was developed to explain the QMO in conventional three-dimensional (3D) metals which put limits on its applicability to the new classes of quasi-twodimensional (Q2D) conductors like the layered organic conductors and superconductors, high- $T_{c}$ cuprates, gra- phite, graphen, GaAs- and Si-based heterostructures, artificial lateral superlattices etc. These conductors display both the $\mathrm{SdH}$ and dHvA oscillations but their shapes often cannot be interpreted in terms of the LK theory. A typical examples are the so-called "forbidden frequencies" in the Fourier spectrum of the QMO and anomalies in the angular and amplitude dependencies of the harmonics in some Q2D conductors. Deviations from the LK behavior in Q2D conductors originate, in particular, from the effects due to the broadening of the sharp Landau levels of the ideal 2D electron gas into Landau bands as shown in Fig. 1. We will demonstrate below that there is a direct relation between the each specific mechanism of the Landau level broadening (or splitting) and the corresponding damping factor. The number of these factors in the Q2D conductors is larger than in the 3D metals. In contrast to the standard LK theory they differ for the thermodynamic dHvA and kinetic SdH oscillations. 


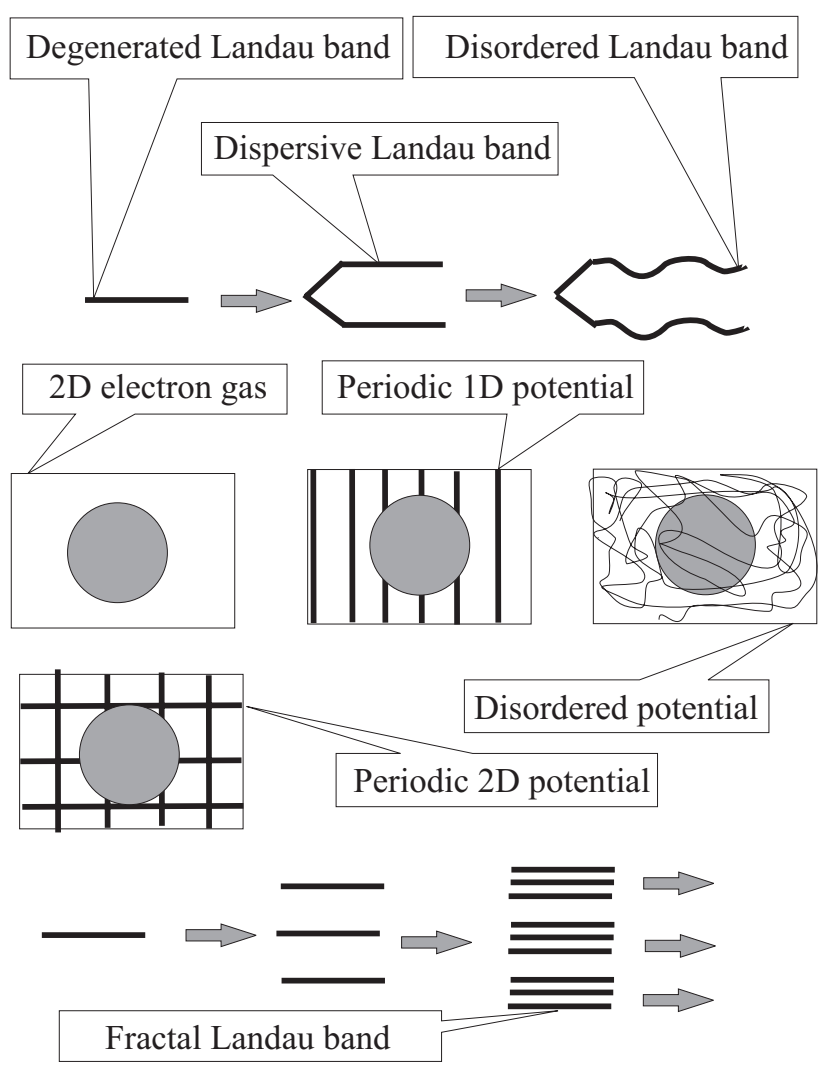

Fig. 1. The Landau bands in $2 \mathrm{D}$ conductors originate from the lifting up the degeneracy of the Landau orbits on a position by the periodic or disordered potential. In case of a $2 \mathrm{D}$ periodic potential the Landau levels split into fractals.

\subsection{The magnetization oscillations in $3 D L K$ theory}

To formulate the problem more precisely, consider first the basic points of the LK approach. The oscillating part of the magnetization in the LK theory can be written as follows:

$$
\tilde{M}(B)=\operatorname{Im} \sum_{p=1}^{\infty} \frac{M_{3 D}}{p^{3 / 2}} \exp \left[2 \pi i p\left(\frac{F}{B}-\gamma\right)\right] R^{L K}(p) .
$$

Here $M_{3 D}$ is the amplitude of the magnetization oscillations. The frequency of the oscillations $F=c S\left(\varepsilon_{F}\right) / 2 \pi e \hbar$ is proportional to the area enclosed by the orbit in the momentum space at the Fermi energy $S\left(\varepsilon_{F}\right)$. The parameter $\gamma$ in the LK theory equals to $1 / 2$, which is equivalent to the factor $(-1)^{p}$ in Eq. (1), but in general, it can deviate from this value. The amplitudes of harmonics are given by the product of the Dingle, $R_{D}(p)$, temperature $R_{T}(p)$, and the LK spin, $R_{S}^{L K}(p)$, factors:

$$
R^{L K}(p)=R_{D}(p) R_{T}(p) R_{S}(p) .
$$

These factors together with the Eq. (1) contain information on the FS shape, effective electron masses, scattering times, and $g$ factors. (i) The period of the $\mathrm{dHvA}$ oscillations in the inverse magnetic field $1 / B$ gives a value of the cross section of the FS, $S\left(\varepsilon_{F}\right)$, by the plane perpendicular to the magnetic field. By changing the magnetic field orientation one can restore the shape of the FS.

(ii) The scattering time, $\tau$, is determined from the Dingle factor which in terms of the Dingle temperature $T_{D}=\hbar / \tau k_{B}$ takes the form

$$
R_{D}(p)=\exp \left(-\frac{p \alpha m^{*} T_{D}}{m_{e} B}\right)
$$

The notations here are $\alpha=2 \pi^{2} m_{e} k_{B} / e \hbar=14.9 \mathrm{~T} / \mathrm{K}$, $k_{B}$ is the Boltzmann constant, and $m_{e}$ is the electron mass. The effective mass of electron is determined by the FS cross-section derivative $m^{*}=(1 / 2 \pi) \partial S(\varepsilon) / \partial \varepsilon$ and depends on the orientation of the magnetic field but not on its strength. The scattering time, therefore, can be obtained from the logarithmic Dingle plot of the $p$ th harmonics amplitude.

(iii) The temperature factor in Eq. (2) is given by

$$
R_{T}(p)=\frac{\lambda p}{\sinh (\lambda p)}
$$

where the parameter $\lambda \equiv 2 \pi^{2} k_{B} T m^{*} / m_{e} \hbar \omega_{c}$ is proportional to the temperature. For the fixed value of the field $B$ the shape of the $R_{T}(p)$ depends only on the effective electron mass. Therefore, the effective mass in the LK theory is determined from the experimental curve for the temperature behavior of the amplitude $R^{L K}(p)$ since other terms in Eq. (1) are temperature-independent.

(iv) The spin-factor in the LK theory is given by

$$
R_{S}(p)=\cos \left(\frac{\pi p g m^{*}}{2 m_{e}}\right)
$$

The effective mass depends on the FS cross section which can be changed by the magnetic field rotation. For the closed orbit $m^{*} \propto 1 / \cos \theta$ where $\theta$ is an angle between the magnetic field and the one of the FS symmetry axis. The spin-factor becomes zero at the angles which satisfy the condition $g m^{*}(\theta) / m_{e}=2 n+1$, where $n$ is an integer. This is the spin-zero effect which makes possible to determine the $g$ factor, provided that the effective mass is known from the temperature factor $R_{T}(p)$.

(v) It is believed in the LK theory that the SdH magnetoresistance oscillations are completely due to the Landau energy spectrum quantization and therefore a simple relation between the oscillation part of the conductivity and the magnetization oscillations holds: $\tilde{\sigma} \propto B^{2} \partial \tilde{M} / \partial B$.

The above scheme works nicely in conventional $3 \mathrm{D}$ metals under the conditions when the chemical potential $\mu$ is fixed at the Fermi energy $\varepsilon_{F}$ which is the largest energy scale in the problem (i.e., much larger than the disorder 
potential, density wave or any other periodic perturbation, temperature, cyclotron frequency etc., which is really the case in conventional 3D metals).

Numerous deviations from the LK theory have been observed in different quasi-two-dimensional (Q2D) conductors. They are caused by the chemical potential oscillations [4-22], magnetic breakdown [4-8,23-29], dispersive and incoherent transport of electrons across the layers [4-10, 30-36], spin- (SDW) and charge-density-waves (CDW) [4-7]. The first observation of the QMO in layered superconductors in 1976 [37] was a surprise since magnetic field penetrates into the bulk of a superconductor in a form of the Abrikosov vortices and it was believed that the QMO are impossible in superconductors. Two decades later experiments confirmed the QMO in the other conventional superconductors [38,39]. The additional damping of the QMO below the upper critical magnetic field in 2D superconductors was explained by the scattering of the quasiparticles on the "vortex-matter" in the superconducting mixed state [39-47]. A review of the theoretical papers and relevant experiments on the $\mathrm{QMO}$ in quasi-2D conventional superconductors was given in Ref. 8. It was revealed later that in layered organic superconductors which display the QMO under the conditions of incoherent electron hopping between the layers [33-35] the amplitudes of the SdH and dHvA oscillations enhance below the upper critical magnetic field as a result of the restoration of the coherence across the layers in the superconducting state [10]. After more than twenty years of intensive researches, a new surprise was the discovery of the QMO in the high- $T_{c}$ cuprates [48]. It gave a new impetus to the studies of a long standing puzzle of the cuprates which are non-Drude quasi-2D strongly correlated conductors with the pseudogap in the energy spectrum. The SdH and dHvA oscillations in cuprates deviate from the LK theory. The mechanisms of these deviations are in part the same as in layered organic superconductors which is not recognized yet in the QMO community. A typical mistake is an application of the thermodynamic set of the damping factors to the kinetic SdH oscillations.

The purpose of this paper is to describe within a unified approach the effects related to the formation of the Landau dispersive and disordered bands on the QMO in layered conductors which are beyond the points (i)-(v) of the standard LK theory. We will focus a special attention on the $\mathrm{SdH}$ oscillations in periodic magnetic breakdown Fermisurface configurations and compare results with the $\mathrm{dHvA}$ and Weiss oscillations.

This paper is devoted to Emanuil Aizikovich Kaner with whom we wrote two works on the oscillations in 2D coherent MB structures [26,27]. We have predicted in [27] a cascade of the magnetic-breakdown structural Pierls-like phase transitions which is similar to recent observations in graphite [7,49].

\section{Quantum magnetic oscillations in quasi-2D conductors}

\subsection{The origin of the Landau bands and the difference between the $S d H, d H v A$ and Weiss oscillations}

The Landau levels in 2D electron gas are flat and degenerated with respect to the Landau orbit center position. The degeneracy is large and equals to $s=\Phi / \Phi_{0}$, where $\Phi$ is the flux through a 2D sample and $\Phi_{0}=h c / e$ is the flux quantum. Any spatial disorder or periodicity within the 2D plane lifts up the degeneracy and broaden Landau levels into the Landau bands which can be either dispersive, or disordered, or fractal, as illustrated in Fig. 1. The fractal splitting of the Landau levels occurs if external potential has periodicity in two directions and the flux through a unit cell is an irrational number in flux units. A fine substructure of the energy levels within the Landau bands in that case is known as the Azbel-Hofstadter butterfly [50-57]. In case of 1D artificially produced periodic potential as well as for the periodic magnetic-breakdown trajectories in the momentum space shown in Fig. 2 the energy spectrum within the $N$ th Landau band is dispersive which means that electron energy is a periodic function of the quasi-momentum $E_{N}(p)=E_{N}(p+\hbar / a)$, where $a$ stands for the spatial period of the trajectory. The width of the dispersive Landau bands is a periodic function of the inverse magnetic field which gives rise to the Weiss (bandwidth) oscillations [58-65]. These oscillations differ from the $\mathrm{SdH}$ or dHvA oscillations. They are less damped at low fields $B<<B_{0}$ and fade away at higher fields. $\left(B_{0}\right.$ is usually less than $0.1-0.5 \mathrm{~T}$ in lateral superlattices and amount to $10-30 \mathrm{~T}$ in layered organic conductors.)
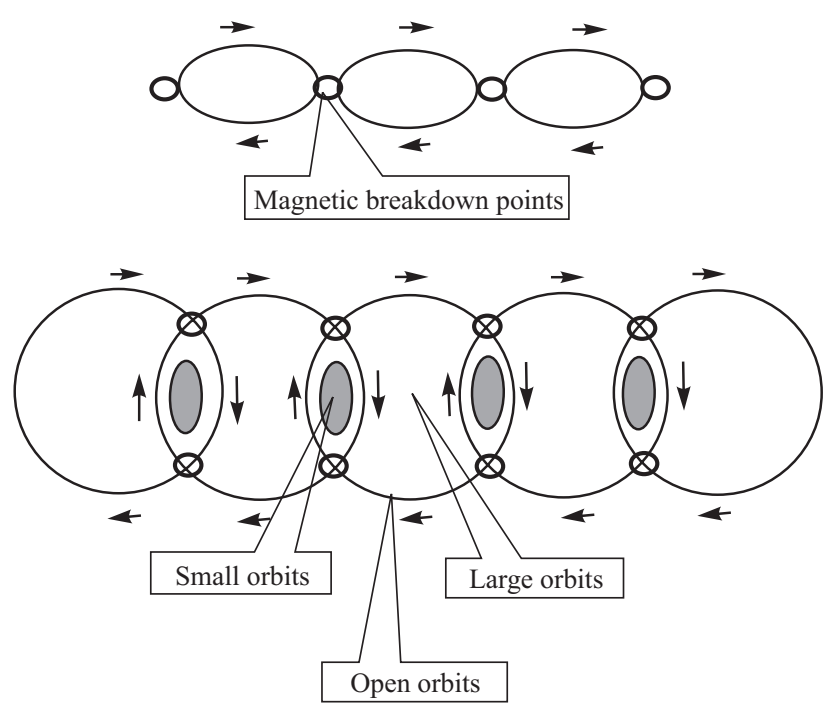

Fig. 2. The Fermi contours composed of a periodic chains of closed orbits connected by the magnetic-breakdown points typical for some quasi-2D organic conductors and artificial lateral superlattices. 
In a regular layered conductors dispersive Landau bands appear due to the electron hopping between the layers. In case of an irregular layer-stacking, or if $t<<\hbar / \tau$, this hopping is incoherent which has a strong impact on the quantum magnetic oscillations of the conductivity across the layers ( $t$ is the interlayer hopping integral, $\tau$ is the scattering time within the layers). The incoherence means that the hopping between the neighboring layers require a time $\hbar / t>>$ so that electrons scatter many times within the layers before successive hopping. The momentum in direction perpendicular to layers is no longer a good quantum number and there is no 3D FS (in other terms, the cylinder warping $t$ is much less than the FS smearing by impurities $\hbar / \tau)$. In such Q2D conductors the QMO are due to the Landau quantization within the planes $[10,36,66]$.

A potential disorder within the planes is especially crucial for the quantum oscillations of the in-plane conductivity. A remarkable example is the $2 \mathrm{D}$ electron gas in which the low-field $\mathrm{SdH}$ oscillations of the diagonal conductivity increase their amplitudes with the increase of magnetic field and transform gradually into sharp peaks at higher fields when the integer quantum Hall effect regime sets in [67-69]. These peaks are centered exactly at the Landau levels where narrow stripes of the delocalized states are placed. The other states within the disorder-broaden Landau bands are localized. The localization, important for the conductivity oscillations, is not so crucial for the thermodynamic dHvA oscillations. Therefore, in sharp contrast to the LK theory developed for the 3D conventional metals, there is a dramatic difference between the $\mathrm{SdH}$ and dHvA oscillations in quasi-2D conductors. In layered conductors the shape of the QMO is determined by the different damping factors, some of which oscillate in inverse magnetic field and have different shape for the $\mathrm{SdH}$ and dHvA oscillations. In conventional 3D metals the chemical potential equals to the Fermi energy which is not the case in quasi$2 \mathrm{D}$ conductors and the chemical potential oscillations is another source of deviations from the LK theory in these materials (see [11-21]) which we do not consider here.

In what follows we derive damping factors which appear as a result of the broadening of the Landau levels into bands and show the difference between the $\mathrm{SdH}$, dHvA and Weiss oscillations. We put a special emphases to calculations of the magnetic-breakdown factors for the Fermi contours shown in Fig. 2 which are typical for different organic conductors and some artificial lateral superlattices.

\subsection{The quantum oscillations of magnetization in quasi-2D conductors}

The quasiclassical quantization rule of the closed 2D orbit encompassing an area $S(E)$ in the momentum space

$$
S(E)=\frac{2 \pi e B \hbar}{c}(n+1 / 2),
$$

yields a periodic set of sharp Landau levels separated by the cyclotron energy $\hbar \omega_{c}$, where the cyclotron frequency $\omega_{c}=e B / m^{*} c$. In case the Landau bands are broaden into bands the energy spectrum takes the form

$$
E_{n}(\xi)=\hbar \omega_{c}(n+1 / 2)+\xi \text {. }
$$

Here $\xi$ is the energy related to additional degree of freedom responsible for the broadening or splitting Landau levels. Let $g(\xi)$ be the density of states (DOS) associated with this degree of freedom. If there are two independent such degrees of freedom described by the variables $\xi_{1}$ and $\xi_{2}$, then $\xi=\xi_{1}+\xi_{2}$, and the total DOS is given by a convolution of the two related densities of state $g_{1}\left(\xi_{1}\right)$ and $g_{2}\left(\xi_{2}\right)$ :

$$
g(\xi)=\int d \omega g_{1}(\xi-\omega) g_{2}(\omega) .
$$

A generalization to the many degrees of freedom is straightforward.

The density of states of the whole system with the energy spectrum $E_{n}(\xi)$ is given by

$$
\rho(\varepsilon, B)=s \int_{-\infty}^{\infty} d \xi g(\xi) \sum_{n=0}^{\infty} \delta\left(\varepsilon-\hbar \omega_{c}(n+1 / 2)-\xi\right) .
$$

The quantity $s=\Phi / \Phi_{0}$ is the degeneracy of the Landau level. Using the Poisson summation formula

$$
\sum_{n=0}^{\infty} f(n+1 / 2)=\int_{0}^{\infty} f(x) d x+2 \operatorname{Re} \sum_{p=1}^{\infty}(-1)^{p} \int_{0}^{\infty} f(x) \mathrm{e}^{2 \pi i p x} d x
$$

one can single out an oscillating part of the $\rho(\varepsilon, B)$, which yields

$$
\tilde{\rho}(\varepsilon, B)=\frac{s}{\hbar \omega_{c}} 2 \operatorname{Re} \sum_{p=1}^{\infty}(-1)^{p} \exp \left(\frac{2 \pi i p \varepsilon}{\hbar \omega_{c}}\right) I(p),
$$

where the factor $I(p)$ is a Fourier transform of the DOS $g(\xi)$

$$
I(p)=\int_{-\infty}^{\infty} d \xi g(\xi) \exp \left(-\frac{2 \pi i p \xi}{\hbar \omega_{c}}\right) .
$$

This universal factor is a generic for the thermodynamic QMO in a sense that all other damping factors of dynamical origin (the Dingle and the spin factors, for example) can be obtained directly from it. (The temperature factor is not a dynamic factor because it comes from the temperature smearing of the Fermi function.)

The magnetization oscillations in the Q2D layered conductors with the DOS (11) is given by

$$
\tilde{M}(B)=\operatorname{Im} \sum_{p=1}^{\infty} \frac{M_{0}}{p} \exp \left[2 \pi i p\left(\frac{F}{B}-\gamma\right)\right] R_{T}(p) I(p) .
$$


The factor $I(p)$ is a Fourier transform of the DOS within the Landau band $g(\xi)$. If a few mechanisms broad Landau levels into a band $\xi$ is a sum of the variables $\xi_{i}$ and $I(p)$ is a product of the factors $I_{i}(p)$ responsible for each mechanism because in this case the total DOS $g(\xi)$ is a convolution of the functions $g\left(\xi_{i}\right)$.

In the next sections we will demonstrate how the different damping factors appear within this approach and will calculate the magnetic-breakdown factors for trajectories shown in Fig. 2.

\subsection{The spin factor and the Dingle factor}

Magnetic field split Landau levels of electrons since they have a spin. The DOS associated with the spin degree of electrons is given by

$$
g_{S}(\xi)=\frac{1}{2}\left[\delta\left(\xi-\varepsilon_{+}\right)+\delta\left(\xi-\varepsilon_{-}\right)\right]
$$

where the spin-split energy of the Landau levels is given by $\varepsilon_{ \pm}=g \mu_{B} S B, \mu_{B}=e \hbar / 2 m_{e} c$ is the Bohr's magneton, and the spin eigenvalue is $S= \pm 1 / 2$. A substitution of this DOS into Eq. (12) gives the spin factor $R_{S}(p)$ of Eq. (5).

Impurities broad Landau levels into disordered Landau bands. To calculate the appropriate DOS $g^{i}(\xi)$ within the Landau bands is not a simple problem. It is generally accepted and confirmed by numerous experiments that a good approximation for the DOS within the impuritybroaden Landau bands $g^{i}(\xi)$ is the Lorentzian

$$
g^{i}(\xi)=\frac{1}{\pi} \frac{\Gamma}{\xi^{2}+\Gamma^{2}} .
$$

The factor Eq. (12) yields in this case a famous exponential Dingle factor $I^{i}(p)=R_{D}(p)$ in the form of Eq. (3).

The function $g^{i}(\xi)$ in general deviates from the simple Lorentzian shape. That is, for example, in a 2D electron gas at high magnetic fields in the integer quantum Hall effect regime. In that regime the DOS within the Landau bands has a narrow stripe of delocalized states in the middle and localized states in the wings which have rather Gaussian than Lorentzian shape. We do not consider the quantum Hall effect here and limit our consideration to the QMO only.

\subsection{The layer-stacking factor}

The electron hopping across the layers broadens Landau levels into the bands. In case of a periodic layer stacking and under the condition of a nearest layer electron hopping the DOS associated with this degree of freedom is

$$
g(\xi)=\frac{1}{\pi}\left(4 t^{2}-\xi^{2}\right)^{-1 / 2}
$$

This function has a square-root singularities at the band edges $\varepsilon_{0}= \pm 2 t$ which yields the following oscillating layer-stacking factor [29]

$$
I(p)=J_{0}\left(\frac{4 \pi t p}{\hbar \omega_{c}}\right),
$$

where $t$ is the hopping integral between the layers and $J_{0}$ is the Bessel function. In the case of a regular periodicity in layer stacking the electron hopping across the layers can be described in terms of the dispersion relation $\varepsilon(q)$. The factor $I(p)$ can be written in that case in the form

$$
I(p)=\int_{-\pi / a}^{\pi / a} \frac{d q a}{2 \pi} \exp \left(-\frac{2 \pi i p \varepsilon(q)}{\hbar \omega_{c}}\right) .
$$

Here $a$ is the distance between the layers. For the nearest layer hopping the dispersion $\varepsilon(q)=2 t \cos (q a)$ and Eq. (18) gives exactly the factor Eq. (17). A description in terms of the dispersion $\varepsilon(q)$, in fact, is an equivalent to the standard approach with a 3D Fermi surface in the shape of a warped cylinder. The oscillations of the Bessel function in Eq. (17) modulate the dHvA oscillations exactly in the same fashion as the so-called "neck and belly" frequencies in the LK approach.

The description of the electron hopping across the layers in terms of the DOS $g(\xi)$ is more general. It does not imply a regularity in the layer stacking and can be applied even if there is no 3D FS and electron hopping across the layers is incoherent and cannot be described by a dispersion relation $\varepsilon(q)$. The absence of the $3 \mathrm{D}$ FS (the interlayer incoherence) was established with the help of the QMO in some layered organic conductors [33-35] and recently in the high- $T_{c}$ cuprates [48]. Long before that a model of the interlayer incoherence was considered in the context of intercalation defects and the corresponding layer-stacking factor (12) was calculated [29]. It was shown that if there is a small concentration $c \ll 1$ of the stacking faults in a sample then $I(p)$ contain exponential Dingle-like terms caused by the disorder in direction across the layers $[29,47]$. The stacking fault means that the local hopping integral, $t_{0}$, for the hopping from the "defect layer" to the nearest-neighbor layers is less than $t$. The layer-stacking factor in that case is given by

$$
I(p)=(1-c) J_{0}\left(\frac{4 \pi t p}{\hbar \omega_{c}}\right)+c \delta I(p),
$$

where

$$
\delta I(p)=\exp \left(-\frac{2 \pi p}{\hbar \omega_{c}} \sqrt{t^{2}-t_{0}^{2}}\right) \cos \left(\frac{4 \pi t_{0} p}{\hbar \omega_{c}}\right) .
$$

The layer-stacking factor $I(p)$ given by Eqs. (19) and (20) is an oscillating function of the inverse magnetic field which modulates the dHvA oscillations. The Dingle-like exponent in Eq. (20) can be written in a standard form 
$\exp \left(-2 \pi / \omega_{c} \tau_{d}\right)$ where the quantity $\tau_{d}=\hbar / \sqrt{t^{2}-t_{0}^{2}}$ is the scattering time associated with the electron hopping across the irregularly stacked conducting planes.

\subsection{The dynamic localization and the tilted magnetic field effects}

The layer-stacking factors (17) and (19) oscillate as a function of the ratio $t / \hbar \omega_{c}$. The frequency of these, and related dHvA, oscillations depend on the hopping integral value. We consider here two ways of effective changing $t$ by external influence.

In layered conductors with a large hopping integrals $t>\hbar / \tau$ the electron transport across the layers is coherent and the 3D FS has a shape of the warped cylinder. If external magnetic field deviates from the cylinder axis at the angle $\Theta$, the hopping integral between the layers is effectively renormalized by the angle-dependent Yamaji factor [30]

$$
t_{\mathrm{eff}}(\Theta)=t J_{0}\left(k_{F} a \tan \Theta\right),
$$

where $J_{0}(x)$ is the Bessel function, and $k_{F}$ stands for the Fermi wave number. A substitution of the $t_{\text {eff }}(\Theta)$ into the layer-stacking factor (17) makes it an oscillating functions of the angle $\Theta$.

Another opportunity is with the dynamic localization which is an effective modulation of $t$ by the resonance between the Bloch-Stark oscillations of the frequency $e E a / \hbar$ with the external electromagnetic wave of the frequency $\omega$ polarized perpendicular to the layers with the electric field $E$ along this direction. As a result, the effective hopping integral takes the form [31,32]

$$
t_{\mathrm{eff}}=t J_{0}\left(\frac{e E a}{\hbar \omega}\right)
$$

Therefore, by tilting the magnetic field and by applying polarized laser irradiation to layered conductor one can effectively change the hopping integral value and thereby the shape of the dHvA oscillations.

\subsection{The thermodynamic magnetic-breakdown factor}

In some organic layered compounds the Fermi counters are composed of periodic set of closed orbits coupled by the magnetic breakdown (MB) as shown in Fig. 2. Consider first a chain in the upper side of this picture. The Landau bands for that chain are dispersive and the energy spectrum within the Landau band is given by [25-27]

$$
\varepsilon(q)=\frac{\hbar \omega_{c}}{\pi} \arcsin (\sqrt{W} \cos q L)
$$

Here $W$ is the probability of the magnetic breakdown,

$$
W=\exp \left(-\frac{B_{0}}{B}\right)
$$

The quantity $B_{0}$ is the magnetic-breakdown field, and $L$ is the spatial period of the "magnetic-breakdown chain" (periodic trajectory in the momentum space composed of the closed orbits with the period of the Brillouin cell size). Substituting the dispersion relation Eq. (23) into Eq. (18) we arrive at the following expression for the magneticbreakdown factor $I(p) \equiv R^{M B}(p)$

$$
R^{M B}(p)=\frac{2}{\pi} \int_{0}^{\pi / 2} d y \cos [2 p \arcsin (\sqrt{W} \cos y)] .
$$

Completing the integration, we have

$$
R^{M B}(p)=1+\sum_{k=1}^{p} \frac{(-1)^{k}}{(k !)^{2}} W^{2 k} \prod_{l=0}^{k-1}\left(p^{2}-l^{2}\right) .
$$

The MB factor as a function of the $0 \leq W \leq 1$ is a polynomial with the fixed values at the boundaries: $R^{M B}(p)=1$ for $W=0$ and $R^{M B}(p)=0$ for $W=1$. For the first three harmonics $p=0,1,2, \ldots$, these polynomials are

$$
R^{M B}(0)=1, R^{M B}(1)=1-W, R^{M B}(2)=1-4 W^{2}+3 W^{4} .
$$

The plot of the $R^{M B}(p)$ is also shown in Fig. 3. The breakdown field, $B_{0}$ in layered organic conductors vary from the few Tesla to few tenth of Tesla. It can be significantly decreased by applying the external pressure or by the suppression of the density waves, in case they are responsible for the opening the MB gap at 2D the FS [4-10].

An important examples of the Fermi contours with the $\mathrm{MB}$ are shown in Fig. 2. Consider first the upper chain in this figure and let us assume that the small circles in a chain are small $\alpha$ orbits with the area inside $S_{\alpha}(\varepsilon) \ll S(\varepsilon)$. In that case the effective MB probability through small orbits, $W_{\text {eff }}$, oscillates in the inverse magnetic field with the amplitude of the order of unity for $W \ll 1$. The $W_{\text {eff }}$ is given by (see [24-26] for details)

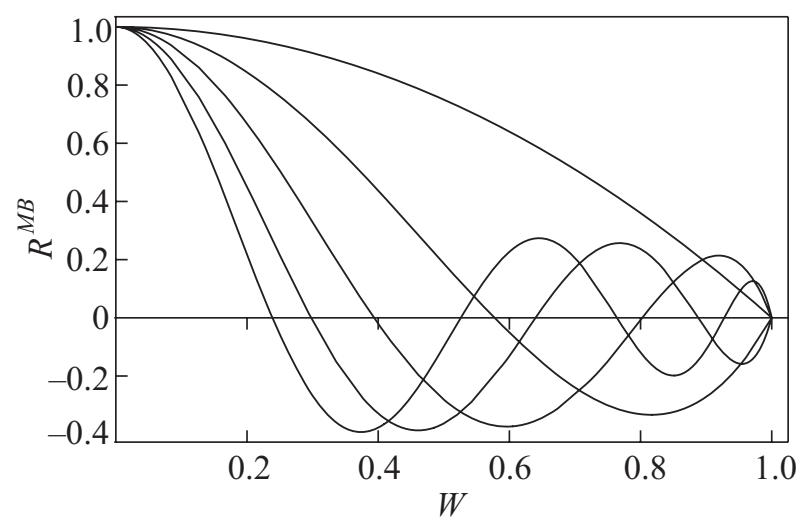

Fig. 3. The magnetic-breakdown factors $R^{M B}(p)$ as a function of the MB probability $W$ for the $p=1,2,3,4,5$. Note that the harmonics integer $p$ exactly equals to the number of zeroth in the polynomial function $R^{M B}(p)$. 


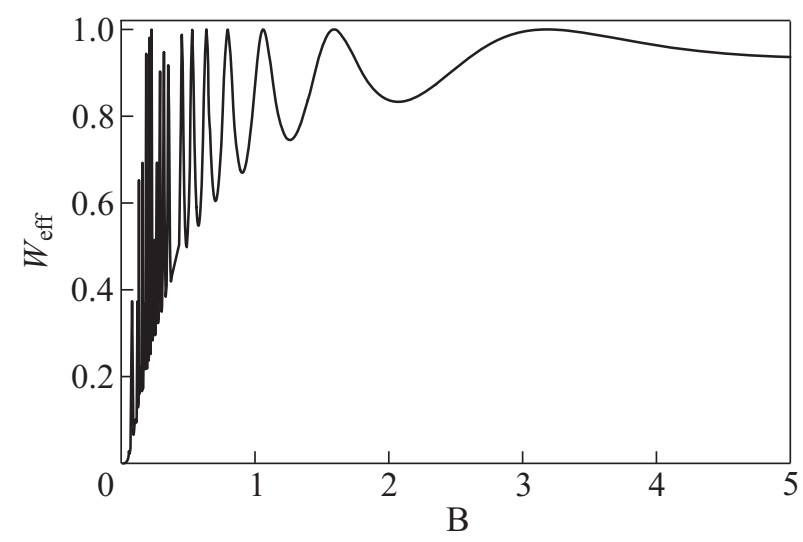

Fig. 4. The oscillations of the effective MB probability given by Eq. (28).

$$
W_{\text {eff }}=\frac{W^{2}}{W^{2}+4(1-W) \sin ^{2} \varphi_{\alpha}} .
$$

This quantity oscillates with the frequency of the closed orbit $\varphi_{\alpha}=\pi F_{\alpha} / B$ which is proportional to the crosssection area of the $\alpha$ orbit in the momentum space at the Fermi energy $S_{\alpha}\left(\varepsilon_{F}\right)$ :

$$
F_{\alpha}=\frac{c S_{\alpha}\left(\varepsilon_{F}\right)}{2 \pi e \hbar} .
$$

The parameter $\gamma$ also should be replaced by the effective quantity $\gamma_{\text {eff }}$ which is an oscillating function of the $\varphi_{\alpha}$ :

$$
\gamma_{\text {eff }}=-\frac{1}{\pi}\left[\arctan \left(\frac{1+\tau^{2}}{1-\tau^{2}} \tan \varphi_{\alpha}\right)-\varphi_{\alpha}\right] \text {. }
$$

The $\gamma_{\text {eff }}$ determines the Landau-band center position which therefore oscillates with the frequency of the closed orbit $F_{\alpha}$. The Landau bandwidth also oscillate with this frequency

$$
\Delta \varepsilon=\frac{2 \hbar \omega_{c}}{\pi} \arcsin \left(\sqrt{W_{\mathrm{eff}}}\right)
$$

These oscillations of the Landau-band spectrum explain naturally the appearance of the so-called forbidden frequencies observed in quantum oscillations in the MB regime of some layered organic conductors. These forbidden frequencies in the Fourier spectrum of the magnetic oscillations imply that electrons change abruptly the sense of rotation in a magnetic field on some sections of the Fermi contour. This paradox is naturally resolved in our approach by the oscillations of the MB factor. More details on the problem of the forbidden frequencies can be found in the papers [4-6,19-22]. The forbidden frequencies also have been observed in the $2 \mathrm{D}$ electron systems with the artificially modulation or lateral superlattices which display the MB Fermi contours like the ones in Fig. 2 but at lower energy scale $[62,63]$. The MB field $B_{0}$ in the $2 \mathrm{D}$ artificial structures is much less than in layered organic ET salts.

\section{The band conductivity of a 2D system in perpendicular magnetic field}

The conductivity of a 2D conductor in perpendicular magnetic field can be written in the following form [66]:

$$
\sigma=\sigma_{0} \operatorname{Re} \sum_{p=-\infty}^{\infty} \mathrm{e}^{i 2 \pi p \gamma} N(p) \int d E A_{p}(E)\left(-\frac{\partial f}{\partial E}\right)
$$

where $\sigma_{0}=e^{2} \Phi /\left(V \Phi_{0} \omega_{c}\right)$ ( $V$ is the volume of a sample). The other quantities in Eq. (32) are

$$
\begin{gathered}
N(p)=\int d \varepsilon g(\varepsilon) v^{2}(\varepsilon) \exp \left(\frac{2 \pi i p \varepsilon}{\hbar \omega_{c}}\right), \\
A_{p}(E)=\exp \left(\frac{2 \pi i p E}{\hbar \omega_{c}}\right) \frac{\tau(E)}{\hbar}\left(1+\frac{2 \pi|p|}{\omega_{c} \tau}\right) R_{D}(p, E) .
\end{gathered}
$$

The generalized Dingle factor here is written in terms of the scattering time $R_{D}(p, E)=\exp \left(-2 \pi|p| / \omega_{c} \tau\right)$ which is defined through a self energy $\hbar / \tau(E)=|\operatorname{Im} \Sigma(E)|$ and therefore depends on the energy $E$.

The important difference of the $\mathrm{SdH}$ series (32) from the dHvA oscillations (13) in 2D conductors is a new damping factor $N(p)$ which differs from the thermodynamic factor $I(p)$ by the velocity squared $v^{2}(\varepsilon)$ under the integral.

In the LK theory the electron velocity at the $3 \mathrm{D} F S$ is assumed to be a constant $v^{2}(\varepsilon) \approx v(0)^{2}$. In this approximation the kinetic factor is proportional to the thermodynamic one $N(p)=v(0)^{2} I(p)$ and because of that the SdH oscillations are reduce to the dHvA oscillations in the LK theory. This is not the case in 2D and layered conductors. We will illustrate that difference in the next sections.

\subsection{The SdH oscillations in layered conductors}

Consider first the $\mathrm{SdH}$ oscillations in a layered conductor with the electron dispersion across the layers $\varepsilon(q)=t \cos (q a / \hbar)$. In terms of dispersion $\varepsilon(q)$ the kinetic layer-stacking factor takes the following form

$$
N_{z z}(p)=\int_{-\pi / a}^{\pi / a} \frac{d q a}{2 \pi} v_{z}^{2}(q) \exp \left(-\frac{2 \pi i p \varepsilon(q)}{\hbar \omega_{c}}\right),
$$

where $v_{z}(q)=\partial \varepsilon(q) / \partial q$ is the electron velocity across the layers. A calculation of the integral yields

$$
N_{z z}(p)=\frac{a^{2} t \omega_{c}}{2 \pi p \hbar} J_{1}\left(\frac{2 \pi p t}{\hbar \omega_{c}}\right) .
$$

The difference with the thermodynamic layer-stacking factor $I(p)=J_{0}\left(2 \pi p t / \hbar \omega_{c}\right)$ is evident. An important point is that for a regular stacking both layer-stacking factors oscillate in inverse magnetic field due to the warping of the Fermi surface. 
In the "tau-approximation" the scattering time does not depend on energy, $\tau(E)=\tau$. In that approximation with the help of equation

$$
\int_{0}^{\infty} d E\left(-\frac{\partial f}{\partial E}\right) \exp \left(\frac{2 \pi i p E}{\hbar \Omega}\right) \approx R_{T}(p) \exp \left(\frac{2 \pi i p \varepsilon_{F}}{\hbar \Omega}\right)
$$

one can rewrite the conductivity in a form more similar to the LK expression

$$
\begin{gathered}
\sigma(B)=\frac{2 \sigma_{0} \tau}{\hbar} \times \\
\times \sum_{p=-\infty}^{\infty} N(p)\left(1+\frac{2 \pi|p|}{\omega_{c} \tau}\right) \cos \left[2 \pi p\left(\frac{F}{B}-\gamma\right)\right] R_{T}(p) R_{D}(p) .
\end{gathered}
$$

Putting here $N_{z z}(p)$ one can calculate the conductivity across the layers $\sigma_{z z}$. If temperature and Dingle factors are small $R_{T}(p) R_{D}(p) \ll 1$ then only first few harmonics are important in the series (38). That case was studied in $[71,72]$. With the factor $N_{z z}(p)(36)$ one can to perform a summation in Eq. (38) only numerically. Fortunately, an analytic consideration is possible in the case of a quantum Hall effect. In that case all states are localized within the Landau band $g(\varepsilon)$ except a narrow stripe in the middle which means that $v^{2}(\varepsilon)=0$ for all energies except those within the stripe at the center of band $(\varepsilon=0)$. Therefore, only a narrow stripe of delocalized states contribute to the factor $N(p)$ which does not depend on the $p$ in that case $N(p) \approx \int d \varepsilon g(\varepsilon) v^{2}(\varepsilon)$. Because of that a sum in Eq. (38) can be completed analytically for the arbitrary shape of the function $g(\varepsilon)$. The two terms in the sum (38) determine the Boltzmann and the "quantum" conductivities. The "quantum term" is proportional to the $2 \pi|p| / \omega_{c} \tau$. It is very important in the quantum Hall regime in which both the Botzmann and the "quantum" conductivities are sharply peaked, but nearly cancel each other between the peaks. More details can be found in the papers $[66,73]$.

\subsection{Coherence versus incoherence in a hopping between the layers}

Of special interest is the case of small $t$ since it means a weak coupling between the layers which was really established in some organic conductors. In case of a small $t \ll \hbar / \tau$ a hopping between the layers is incoherent even if a stacking is regular and the layered conductor has no 3D FS, but within the layers a 2D FS survive and the Landau quantization of the 2D electron orbits makes an overall oscillation picture more complex than in a pure $2 \mathrm{D}$ case.

That difference is especially important in layered superconductors with the incoherent electron hopping between the layers in a normal state [10]. The coherence restores in a superconducting state because the hopping integrals are effectively renormalized $t \rightarrow t_{\text {eff }} \approx t+\Delta^{2} / t \approx \Delta^{2} / t \gg t$ by the order parameter $\Delta$ [74]. The hopping between the layers take less time $\hbar / t_{\text {eff }}$ and electrons spend less time within the layers. Correspondingly they less scatter on impurities within the planes which enhances both the kinetic $N_{z z}(p)$ and thermodynamic $I(p)$ layer-stacking factors [10]. If this enhancement is larger than the damping due to the scattering on a "vortex matter" within the layers, than the $\mathrm{SdH}$ and $\mathrm{dHvA}$ amplitudes enhance at fields just below the upper critical one. At first glance that is counter intuitive because the superconductivity is an antagonist of the magnetization and suppresses the QMO in quasi-2D superconductors. This suppression in $2 \mathrm{D}$ case was considered theoretically in a number of papers reviewed in [8] but all these theories ignored the hopping between the layers which is incorrect in general and, as we see, can changes things notably.

To illustrate this, consider a limiting case of a strong incoherence $t \ll \hbar / \tau$ resulting in a localization of electrons in direction perpendicular to layers which means $N_{z z}(p)=0$. A restoration of the coherence in direction perpendicular to the layers makes the factor $N_{z z}(p)$ and the conductivity $\sigma_{z z}$ nonzero. Therefore, the interlayer incoherence $t \ll \hbar / \tau$ is crucial for the anomalous enhancement of the quantum magnetic oscillations of the conductivity $\sigma_{z z}$ in the superconducting state. This effect has been observed in a layered organic conductor $\beta^{\prime \prime}$ in which an incoherent electron transport in the normal state is due to the very small hopping integral between the layers and 3D Fermi surface is absent [10].

The dynamic localization and deviation of external magnetic field from the axis of a $3 \mathrm{D}$ cylinder FS, as was discussed above, also renormalize the hopping integrals. The corresponding effective hopping integrals are given by Eqs. (21) and (22). In both cases, because of the oscillations of the Bessel function, the effective hopping integrals become zero when either $k_{F} a \tan \Theta$ or $e E a / \hbar \omega$ are equal to one of the zeros of the $J_{0}\left(x_{l}\right)=0$. Nearby these values $t_{\text {eff }}$ is small and electron hopping becomes incoherent if $t_{\text {eff }} \ll \hbar / \tau$.

Therefore, by tilting the magnetic field towards the planes and by applying polarized laser irradiation to layered conductor one can effectively change the hopping integral value and thereby switch coherent hopping to the incoherent regime. All this makes a picture of oscillations very complex and require a separate analysis.

\subsection{The magnetic breakdown factor in the $\mathrm{SdH}$ oscillations}

In this section we calculate the factor $N(p)$ in the case when the dispersive Landau bands appear as a result of coherent magnetic breakdown in 2D conductor.

A typical examples are shown in Fig. 2. Consider first a chain of closed orbits in the top of this picture. The energy spectrum within the Landau band in this case is given by Eq. (23). The corresponding kinetic factor takes the form 


$$
N(p)=\left(\frac{\omega_{c} L}{\pi}\right)^{2} M(W, p)
$$

where

$$
M(W, p)=\frac{2}{\pi} \int_{0}^{\pi / 2} d x \frac{W^{2} \sin ^{2} x}{1-W^{2} \cos ^{2} x} \cos [2 p \arcsin (W \cos x)]
$$

After the integration, we have

$$
\begin{gathered}
M(W, 1)=-1+W^{2}+\sqrt{1-W^{2}}, \\
M(W, 2)=1-W^{4}-\sqrt{1-W^{2}} \\
M(W, 3)=-1+W^{2}-2 W^{4}+2 W^{6}+\sqrt{1-W^{2}}, \\
M(W, 4)=1-4 W^{4}+8 W^{6}-5 W^{8}-\sqrt{1-W^{2}}, \\
M(W, 5)= \\
=-1+W^{2}-6 W^{4}+22 W^{6}-30 W^{8}+14 W^{10}+\sqrt{1-W^{2}} .
\end{gathered}
$$

Note that $M(0, p)=M(1, p)=0$ for any harmonics number $p$. This means that the band $\mathrm{SdH}$ conductivity oscillations vanishes in cases of a flat and wide Landau bands, i.e., when the band velocity is zero or if there are no gaps between the Landau bands. The factor $M(W, p)$ for the first five harmonics is shown in Fig. 5. As a function of the MB probability $W$ the function $M(W, p)$ is a sum of the polynomial of the degree $2 p$ and the term $(-1)^{p+1} \sqrt{1-W^{2}}$. The polynomials oscillate within the interval $0 \leq W \leq 1$. Because of these oscillations amplitudes of the higher harmonics at some intervals of magnetic field exceed the amplitudes with the lower $p$. Such unusual behavior of the QMO have been observed in the Fourier spectra of many quasi-2D organic compounds [4-6].

A generalization of the above results to the Fermicontour shown in the lower side of the Fig. 2 is straightforward. Denoting small and large orbits correspondingly by the indices $\alpha$ and $\beta$ one can write the quan-

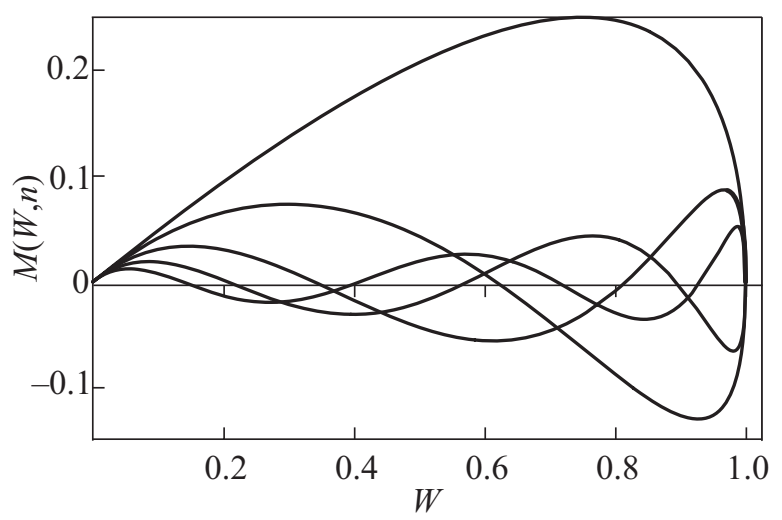

Fig. 5. The damping factor $M(W, n)$ for $n=1,2,3,4,5$ (from top to bottom). tization rules for the corresponding two Landau bands in the form

$$
\begin{aligned}
& S_{\alpha}=\frac{2 \pi e B \hbar}{c}\left[(n+1 / 2)+\frac{(-1)^{n}}{\pi} \arcsin \left(W_{\alpha} \cos q L\right)\right], \\
& S_{\beta}=\frac{2 \pi e B \hbar}{c}\left[\left(n+\gamma_{\mathrm{eff}}\right)+\frac{(-1)^{n}}{\pi} \arcsin \left(W_{\beta} \cos q L\right)\right] .
\end{aligned}
$$

Here $S_{\alpha}(E)$ and $S_{\beta}(E)$ are the cross-section areas enclosed by the $\alpha$ and $\beta$ orbits in the momentum space. The quantum amplitude $W_{\beta}=\left(1-W_{\text {eff }}\right)^{1 / 2}$ is responsible for the resonant MB tunnelling between the $\beta$ orbits through small $\alpha$ orbits and where $W_{\alpha}=W$.

The quantization rules for the $\alpha$ and $\beta$ orbits relate the energy $E$ with the quantum number $n$ and quasi-wavevector $q$, describing the electron dispersion within the Landau bands. To simplify calculations we assume that both orbits are circles in shape with the areas inside correspondingly equal to $S_{\alpha}(E)=2 \pi m_{\alpha} E$ and $S_{\beta}(E)=2 \pi m_{\beta} E$. Then the Landau spectrum can be written in the form of Eq. (23) with the dispersion relations of electrons within the $\alpha$ and $\beta$ Landau bands given by

$$
\varepsilon_{\alpha(\beta)}(q)=\frac{\hbar \omega_{c}^{\alpha(\beta)}}{\pi} \arcsin \left(W_{\alpha(\beta)} \cos q L\right) .
$$

The cyclotron frequencies differs for two Landau bands because of the difference in the effective masses $\omega_{c}^{\alpha(\beta)}=e B / m_{\alpha(\beta)} c$. The coefficient $\gamma_{\text {eff }}$ determines the Landau bands position oscillations which are important for the $\mathrm{SdH}$ and dHvA oscillations but not for the Weiss oscillations stemming from the Landau bandwidth oscillations

$$
N^{\alpha(\beta)}(p)=\left(\frac{\omega_{c}^{\alpha(\beta)} L}{\pi}\right)^{2} M\left(W_{\alpha(\beta)}, p\right) .
$$

The total conductivity is a sum $\sigma=\sigma_{\alpha}+\sigma_{\beta}$. The conductivities here $\sigma_{\alpha(\beta)}$ are given by the series of Eq. (38). The QMO of the total conductivity is a mixture of oscillations with the $\alpha$ and $\beta$ frequencies some of which are "forbidden" in the quasiclassical LK theory because they do not correspond to any of possible classical pathways.

\subsection{The bandwidth oscillations and the Weiss oscillations}

Another distinction from the LK theory is that the $\beta$ bandwidth and corresponding electron velocity oscillate periodically in the inverse magnetic field together with the effective probability $W_{\text {eff }}$. These bandwidth oscillations hold even for the $p=0$ (zero harmonic). The bandwidth conductivity is given by the $p=0$ term in a series (38)

$$
\sigma_{\beta}(p=0)=\frac{\sigma_{0} \tau}{\hbar} M\left(W_{\beta}, 0\right) .
$$


The amplitude of the bandwidth conductivity oscillation is not dumped by the Dingle or temperature factors. It is determined only by the factor

$$
M\left(W_{\beta}, 0\right)=W_{\beta}-1+\sqrt{1-W_{\beta}},
$$

which oscillates in the inverse magnetic field together with the effective probability (28) shown in Fig. 4 because $W_{\beta}=\left(1-W_{\text {eff }}\right)^{1 / 2}$. For high fields, exceeding the magnetic breakdown field $B \gg B_{0}$, the $W_{\text {eff }} \rightarrow 1$ and does not oscillate which means that the magnetic breakdown restores the large $\beta$ orbits completely and small $\alpha$ orbits do not contribute into the QMO. The bandwidth oscillations also vanish if $W \rightarrow 0$.

In $1 \mathrm{D}$ periodically modulated $2 \mathrm{D}$ electron gas a Fermi surface becomes periodic and consisting of the small $\alpha$ and large $\beta$ orbits shown in Fig. 2 [62,63]. The difference with the organic layered compounds is a much larger spatial period of the artificial lateral superlattices resulting in a lower Fermi energies and lower magnetic-breakdown fields. The corresponding bandwidth oscillations in 1D lateral superlattices are known as the Weiss oscillations which hold at low fields (usually less than $0.5 \mathrm{~T}$ ) and vanish at higher fields because the breakdown field $B_{0}$ for the artificial lateral superlattices is usually very small $0.1-0.5 \mathrm{~T}$. The amplitude of the Weiss oscillations is proportional to the $M\left(W_{\text {eff }}, 0\right)$ (47) in which effective probability is given by Eq. (28) (see $[64,65]$ for more details). In layered organic conductors $B_{0}$ is much higher (of the order 10-30 T) and the observation of the MB effect require much stronger magnetic fields.

\section{Conclusions}

In conclusion we will sum up the main results of this paper and discuss briefly another sources of the deviations of the QMO in Q2D conductors from the predictions of the LK theory which have not been touched in the above consideration.

(i) The main conclusion is that the broadening of the sharp and degenerated Landau levels into dispersive or disordered bands results in a specific dumping factors $I(p)(12)$ and $N(p)$ (33) different for the thermodynamic dHvA and kinetic $\mathrm{SdH}$ oscillations. That difference is because the factor $I(p)$ depends only on the DOS within the Landau band $g(\varepsilon)$ while the factor $N(p)$ depends yet on the electron velocity squared $v(\varepsilon)^{2}$ which equals zero for the localized states. The dependence of the kinetic factor on the electron velocity is especially important in layered and quasi-2D conductors in which electron hopping between the layers is incoherent and there is no 3D Fermi surface. Another example is the quantum Hall effect in which electrons are partially localized within the disordered Landau bands.

(ii) In isotropic $3 \mathrm{D}$ conventional metals the electron velocity can be approximated by a constant $v^{2}(\varepsilon) \approx v(0)^{2}$ and the kinetic factor is proportional to the thermodynamic one $N(p)=v(0)^{2} I(p)$. In that case, as in LK theory, the $\mathrm{dHvA}$ and $\mathrm{SdH}$ oscillations have the same damping factors which can be obtained from the thermodynamic factor $I(p)$ as explained in the Introduction.

(iii) The layer-stacking factors related to the (coherent or incoherent) electron hopping between the layers are absent in the LK theory. For a simple cosine dispersion in direction perpendicular to the layers they can be expressed in terms of the Bessel functions (see Eqs. (17) and (36)) oscillating in perpendicular magnetic field. The layer-stacking factors $I(p)$ and $N(p)$ depend on the hopping integrals which can be effectively changed by the tilting magnetic field towards the layers (see Eq. (21)) or due to the dynamic localization in the polarized high-frequency electromagnetic radiation (see Eq. (22)). These effective hopping integrals are also oscillating functions of their arguments and because of that electron hopping becomes incoherent, if $t_{\text {eff }} \ll \hbar / \tau$, even in a case of periodic stacking.

(iv) Another example illustrating importance of the layer-factors is the anomalous enhancement of the QMO below the upper critical magnetic field which holds in layered conductors with small $t \rightarrow 0$ and incoherent hopping between the layers. A restoration of the interlayer coherence in the superconducting state results in an anomalous enhancement of the $\mathrm{SdH}$ and $\mathrm{dHvA}$ oscillations due to the effective renormalization of the hopping integrals in the superconducting state. In some organic conductors this enhancement exceeds the damping of the QMO by the vortices. This effect is absent in superconductors with a coherent transport of electrons across the layers in the normal state.

(v) In case of coherent magnetic breakdown the thermodynamic factor $I(p)$ is a polynomial of the degree $p$ with respect to the $\mathrm{MB}$ probability $W$ described by Eqs. (25)-(27) and shown in Fig. 3. At fields $B<B_{0}$ the effective probability through a small orbit $W_{\text {eff }}$ oscillate between 0 and 1 with the frequency determined by the small $\alpha$ orbit as shown in Fig. 4.

(vi) The kinetic factor $M(W, p)$, responsible for the $\mathrm{SdH}$ amplitudes, as a function of $W$ (and $W_{\text {eff }}$ ) also oscillates having the $p-1$ zeros within the interval $0<W<1$ as one can see in Fig. 5 and from Eqs. (25)-(27).

(vii) The bandwidth oscillations and the Weiss oscillations are special types of quantum oscillations which vanish at high fields $B \gg B_{0}$. They contribute into the conductivity oscillations beginning from the "zero term" $(p=0)$ given by Eq. (46). The difference between the Weiss and the bandwidth oscillations is as follows. In case of the Weiss oscillations the low-scale Fermi surface originates from the $1 \mathrm{D}$ artificial lateral modulation and has a small MB field $B_{0}$ (usually $0.1-0.5 \mathrm{~T}$ ). In case of layered organic conductors the Fermi energy is much larger and the MB field is about $10-30 \mathrm{~T}$. 
(viii) We do not considered in this article the chemical potential oscillations which is yet another source of deviations from the LK theory in which the chemical potential is fixed at the Fermi energy of 3D metals. This problem is beyond the scope of the present paper. The chemical potential oscillations are thermodynamic in nature. They have the same shape as the magnetization oscillations given by Eq. (13) and depend on the Landau bandwidth through the factor $I(p)$ which was studied above. More details can be found in [11-22] and references therein.

(ix) There is yet another effect responsible for the oscillations of the magnetization and conductivity with the period of the QMO. This effect was predicted in our paper with E. Kaner which we called the magnetic-breakdown Pierls transition [27]. It occurs periodically in inverse magnetic field as a structural phase transition in a halffilled dispersive Landau band [28]. The critical temperature increases proportionally to the MB probability $W(B)$ together with the bandwidth $T_{c} \propto \exp \left(-B_{0} / B\right)$. The structural phase transitions with such typical for the MB exponential form of the $T_{c}(B)$ has been observed recently in graphite $[7,49]$.

\section{Acknowledgments}

This work was supported in part by European Commission CORDIS Seven Framework Program, Project No. 247556.

1. I.M. Lifshitz and A.M. Kosevich, Zh. Eksp. Teor. Fiz. 29, 730 (1956).

2. D. Shoenberg, Magnetic Oscillations in Metals, Cambridge University Press, Cambridge (1984).

3. A.A. Abrikosov, Fundamentals of Theory of Metals, Nauka, Moscow (1987) (in Russian).

4. J. Singleton, Rep. Prog. Phys. 63, 1111 (2000).

5. M.V. Kartsovnik, Chem. Rev. 104, 5737 (2004).

6. J. Wosnitza, Fermi Surfaces of Low-Dimensional Organic Metals and Superconductors, Springer, Berlin (1996).

7. J.S. Brooks, Rep. Prog. Phys. 71, 126501 (2008).

8. T. Maniv, I.D. Vagner, P. Wyder, and V. Zhuravlev, Rev. Mod. Phys. B73, 867 (2001).

9. M.V. Kartsovnik and V.G. Peschansky, Fiz. Nizk. Temp. 31, 249 (2005) [Low Temp. Phys. 31, 185 (2005)].

10. J. Wosnitza, J. Hagel, O. Ignatchik, B. Bergk, V.M. Gvozdikov, J.A. Schlueter, R.W. Winter, and G.L. Gard, J. Low Temp. Phys. 142, 327 (2006); V.M. Gvozdikov and J. Wosnitza, Physica B359-361, 614 (2005); Fiz. Nizk. Temp. 32, 152 (2006) [Low. Temp. Phys. 32, 109 (2006)].

11. D. Shoenberg, J. Low Temp. Phys. 56, 417 (1984).

12. I.D. Vagner, T. Maniv, and E. Ehrenfreund, Phys. Rev. Lett. 51, 1700 (1983); K. Jauregui, V.I. Marchenko, and I.D. Vagner, Phys. Rev. B41, 12922 (1990); M.A. Itskovsky, T. Maniv, and I.D. Vagner, Z. Phys. B: Condens. Matter 101, 13 (1996).

13. M. Nakano, J. Phys. Soc. Jpn. 66, 19 (1997).
14. A.S. Alexandrov and A.M. Bratkovsky, Phys. Rev. Lett. 76, 1308 (1986).

15. P.D. Grigoriev and I.D. Vagner, Pis'ma Zh. Exp. Teor. Fiz. 69, 139 (1999) [JETP Lett. 29, 156 (1999)].

16. P. Grigoriev, Zh. Exp. Teor. Fiz. 119, 1257 (2001) [JETP 92, 1090 (2001)].

17. T. Champel, Phys. Rev. B64, 054407 (2001); T. Champel and V.P. Mineev, Philos. Mag. B81, 55 (2001).

18. V.M. Gvozdikov, A.G.M. Jansen, D.I. Pesin, I.D. Vagner, and P. Wyder, Phys. Rev. B68, 155107 (2003).

19. V.M. Gvozdikov, Yu.V. Pershin, E. Steep, A.G.M. Jansen, and P. Wyder, Phys. Rev. B65, 1651 (2002).

20. J. Wosnitza, V.M. Gvozdikov, J. Hagel, O. Ignatchik, B. Berk, P.J. Meeson, J.A. Shlueter, H. Davis, R.W. Winter, and G.L. Gard, New J. Phys. 10, 083032 (2008).

21. M.A. Itskovsky and T. Maniv, Phys. Rev. B72, 075124 (2005).

22. F.A. Meyer, E. Steep, W. Biberacher, P. Christ, A. Lerf, A.G.M. Jansen, W. Joss, and P. Wyder, Europhys. Lett. 32, 681 (1995); S. Uji, M. Chaparala, S. Hill, P.S. Sandhu, J. Qualls, L. Seger, and J.S. Brooks, Synth. Met. 85, 1573 (1997); E. Steep, L.H. Nguyen, W. Biberacher, H. Muller, A.G.M. Jansen, and P. Wyder, Physica B259-261, 1079 (1999); C.P. Heidmann, H. Mueller, W. Biberacher, K. Neumaier, C. Probst, K. Andres, A.G.M. Jansen, and W. Joss, Synth. Met. 41-43, 2029 (1991); T. Sasaki, H. Sato, and N. Toyota, Solid State Commun. 76, 507 (1990); J. Caulfield, J. Singleton, F.L. Pratt, M. Doporto, W. Lubczynski, W. Hayes, M. Kurmoo, P. Day, P.T.J. Hendriks, and J.A.A.J. Perenboom, Synth. Met. 61, 63 (1993); M.V. Kartsovnik, G.Yu. Logvenov, T. Ishiguro, W. Biberacher, H. Anzai, and N.D. Kushch, Phys. Rev. Lett. 77, 2530 (1996); N. Harrison, J. Caulfield, J. Singleton, P.H.P. Reinders, F. Herlach, W. Hayes, M. Kurmoo, and P. Day, J. Phys.: Condens. Matter 8, 5415 (1996); E. Steep, L.H. Nguyen, W. Biberacher, H. Muller, A.G.M. Jansen, and P. Wyder, Physica B259-261, 1079 (1999).

23. R.W. Stark and L.M. Falicov, Progr. Low Temp. Phys. 5, 235 (1967).

24. M.I. Kaganov and A.A. Slutskin, Phys. Rep. 4, 189 (1983).

25. V.M. Gvozdikov, Fiz. Nizk. Temp. 12, 705 (1986) [Sov. J. Low Temp. Phys. 12, 399 (1986)].

26. V.M. Gvozdikov and E.A. Kaner, Fiz. Nizk. Temp. 13, 59 (1987) [Sov. J. Low Temp. Phys. 13, 32 (1987)].

27. V.M. Gvozdikov and E.A. Kaner, JETP Lett. 43, 570 (1986) [Pis'ma Zh. Exp. Teor. Fiz. 43, 393 (1986)].

28. V.M. Gvozdikov, Fiz. Nizk. Temp. 18, 997 (1992) [Low Temp. Phys. 18, 700 (1992)].

29. V.M. Gvozdikov, Fiz. Tverd. Tela 26, 2574 (1984) [Sov. Phys. Solid State 26, 1560 (1984)].

30. K.Yamaji, J. Phys. Soc. Jpn. 58, 1520 (1989).

31. D.H. Dunlap and V.M. Kenkre, Phys. Lett. A127, 438 (1988).

32. V.M. Gvozdikov, Fiz. Nizk. Temp. 18, 1128 (1992) [Low Temp. Phys. 18, 790 (1992)].

33. J. Wosnitza, S. Wanka, J. Hagel, H. v. Löhneysen, J.S. Qualls, J.S. Brooks, E. Balthes, J.A. Schlueter, U. Geiser, J. Mohtasham, R.W. Winter, and G.L. Gard, Phys. Rev. Lett. 86, 508 (2001). 
34. M.S. Nam, A. Ardavan, J.A. Symington, J. Singleton, N. Harison, C.H. Mielke, J.A. Schlueter, R.W. Winter, and G.L. Gard, Phys. Rev. Lett. 87, 117001 (2001).

35. J. Wosnitza, J. Hagel, P.J. Meeson, D. Bintley, J.A. Schlueter, J. Mohtasham, R.W. Winter, and G.L. Gard, Phys. Rev. B67, 060504(R) (2003); J. Wosnitza, J. Hagel, J.S. Qualls, J.S. Brooks, E. Balthes, D. Schweitzer, J.A. Schlueter, U. Geiser, J. Mohtasham, R.W. Winter, and G.L. Gard, Phys. Rev. B65, 180506(R) (2002).

36. R.H. McKenzie and P. Moses, Phys. Rev. Lett. 81, 4492 (1998); P. Moses and R.H. McKenzie, Phys. Rev. B60, 7998 (1999).

37. J.E. Graebner and M. Robbins, Phys. Rev. Lett. 36, 422 (1976).

38. G. Goll, M. Heinecke, A.G.M. Jansen, W. Joss, L. Nguen, E. Steep, K. Winzer, and P. Wyder, Phys. Rev. B53, 8871 (1996).

39. T. Terashima, C. Haworth, H. Takeya, S. Uji, and H. Aoki, Phys. Rev. B56, 5120 (1997).

40. T. Maniv and I.D. Vagner, Phys. Rev. B38, 6301 (1988).

41. K. Maki, Phys. Rev. B44, 2861 (1991).

42. M.J. Stephen, Phys. Rev. B45, 5481 (1992).

43. A. Wasserman and M. Springford, Physica B194-196, 1801 (1994).

44. M.R. Norman and A.H. MacDonald, Phys. Rev. B54, 4239 (1996).

45. Z. Tesanovich and P.D. Sacramento, Phys. Rev. Lett. 80, 1521 (1998).

46. L.P. Gorkov and J.P. Schrieffer, Phys. Rev. Lett. 80, 3360 (1998).

47. V.M. Gvozdikov and M.V. Gvozdikova, Phys. Rev. B58, 8716 (1998).

48. N. Doiron-Leyraud, C. Proust, D. LeBoeuf, J. Levallois, B. Bonnemaison, R.X. Liang, D.A. Bonn, and L. Taillefer, Nature 447, 565 (2007); D. LeBoeuf, N. Doiron-Leyraud, J. Levallois, R. Daou, J.B. Bonnemaison, N.E. Hussey, L. Balicas, B.J. Ramshaw, R.X. Liang, D.A. Bonn, W.N. Hardy, S. Adachi, C. Proust, and L. Taillefer, Nature 450, 533 (2007); C. Jaudet, D. Vignolles, A. Audouard, J. Levallois, D. LeBoeuf, N. Doiron-Leyraud, B. Vignolle, M. Nar-done, A. Zitouni, R. Liang, D.A. Bonn, W.N. Hardy, L. Taillefer, and C. Proust, Phys. Rev. Lett. 100, 187005 (2008); S.E. Sebastian, N. Harrison, E. Palm, T.P. Murphy, C.H. Mielke, R.X. Liang, D.A. Bonn, and W.N. Hardy, and G.G. Lonzarich, Nature 454, 200 (2008); A. Audouard, C. Jaudet, D. Vignolles, R.X. Liang, D.A. Bonn, W.N. Hardy, L. Taillefer, and C. Proust, Phys. Rev. Lett. 103, 157003 (2009); S.E. Sebastian, N. Harrison, C.H. Mielke, R.X. Liang, D.A. Bonn, W.N. Hardy, and G.G. Lonzarich, Phys. Rev. Lett. 103, 256405 (2009); S.E. Sebastian, N. Harrison, M.M. Altarawneh, R.X. Liang, D.A. Bonn, W.N. Hardy, and G.G. Lonzarich, Phys. Rev. B81, 140505 (2010); J. Singleton, C. de la Cruz, R.D. McDonald, S.L. Li, M. Altarawneh, P. Goddard, I. Franke, D. Rickel, C.H. Mielke, X.Yao, and P.C. Dai, Phys. Rev. Lett. 104, 086403 (2010); S.E. Sebastian, N. Harrison, M.M. Altarawneh, C.H. Mielke, R.X. Liang, D.A. Bonn, W.N. Hardy, and G.G. Lonzarich, Proc. Nat. Acad. Sci. USA 107, 6175 (2010); S.E. Sebastian, N. Harrison, P.A.
Goddard, M.M. Altarawneh, C.H. Mielke, R.X. Liang, D.A. Bonn, W.N. Hardy, O.K. Andersen, and G.G. Lonzarich, Phys. Rev. B81, 214524 (2010).

49. H. Yaguchi and J. Singleton, J. Phys.: Condens. Matter 21, 344207 (2009).

50. M.Ya. Azbel, Zh. Eksp. Teor. Fiz. 46, 730 (1964) [Sov. Phys.-JETP 19, 634 (1964)].

51. D. Hofstadter, Phys. Rev. B14, 2239 (1976).

52. D. Pfannkuche and R. Gerhardts, Phys. Rev. B46, 12606 (1992).

53. D. Springsguth, R. Ketzmerick, and T. Geisel, Phys. Rev. B56, 2036 (1997).

54. M. Taut, H. Eschrig, and M. Richter, Phys. Rev. B72, 165304 (2005).

55. V.M. Gvozdikov and M. Taut, Phys. Rev. B75, 155436 (2007).

56. V.M. Gvozdikov and M. Taut, New J. Phys. 11, 063005 (2009).

57. V.M. Gvozdikov, Phys. Rev. B53, 10133 (1996); J. Phys.: Condens. Matter 6, 6245 (1994).

58. D. Weiss, K. v. Klitzing, K. Ploog, and G. Weimann, Europhys. Lett. 8, 179 (1989); R.R. Gerhardts, D. Weiss, and K.V. Klitzing, Phys. Rev. Lett. 62, 1173 (1989); R.W. Winkler, J.P. Kotthaus, and K. Ploog, ibid. 62, 1177 (1989); P.H. Beton, P.C. Main, M. Davison, M. Dellow, R.P. Taylor, E.S. Alves, L. Eaves, S.P. Beaumont, and C.D.W. Wilkinson, Phys. Rev. B42, 9689 (1990).

59. C.W.J. Beenakker, Phys. Rev. Lett. 62, 2020 (1989).

60. C. Zhang and R.R. Gerhardts, Phys. Rev. B41, 12850 (1990); P. Streda and A.H. MacDonald, Phys. Rev. 41, 11892 (1990); R.R. Gerhardts, Phys. Rev. B45, 3449 (1992).

61. F.M. Peeters and P. Vasilopoulos, Phys. Rev. B46, 4667 (1992).

62. R.A. Deutschmann, W. Wegscheider, M. Rother, M. Bichler, G. Abstreiter, C. Albrecht, and J.H. Smet, Phys. Rev. Lett. 86, 1857 (2001).

63. C. Albrecht, J.H. Smet, D. Weiss, K. von Klitzing, R. Hennig, M. Langenbuch, M. Suhrke, U. Rössler, V. Umansky, and H. Schweizer, Phys. Rev. Lett. 83, 2234 (1999); M.C. Geisler, J.H. Smet, V. Umansky, K. von Klitzing, B. Naundorf, R. Ketzmerick, and H. Schweizer, Phys. Rev. Lett. 92, 256801 (2004).

64. V.M. Gvozdikov, Phys. Rev. B72, 075135 (2005).

65. V.M. Gvozdikov, Phys. Rev. B75, 115463 (2007).

66. V.M. Gvozdikov, Phys. Rev. B76, 235125 (2007); ibid. B70, 085113 (2004).

67. The Quantum Hall Effect, R.E. Prange and S.M. Girvin (eds.), Springer (1987).

68. D. Yashioka, The Quantum Hall Effect, Springer (2000).

69. Z.F. Ezawa, Quantum Hall Effects, World Scientific (2000).

70. G. Xiong, Shi-Dong Wang, Q. Niu, Yupeng Wang, and X.R. Wang, Europhys. Lett. 82, 47008 (2008).

71. P.D. Grigoriev, Phys. Rev. B67, 144401 (2003).

72. A.S. Alexandrov and V.V. Kabanov, Phys. Rev. Lett. 95, 076601 (2005); ibid. 95, 169902 (2005); Phys. Rev. B76, 233101 (2007).

73. V.M. Gvozdikov, Fiz. Nizk. Temp. 32, 152 (2006) [Low Temp. Phys. 32, 109 (2006)].

74. V.M. Gvozdikov, Fiz. Nizk. Temp. 14, 15 (1988) [Sov. J. Low Temp. Phys. 14, 7 (1988)]. 